\title{
Applicability of A1-term Method to the Scattering by Irregularly Shaped Interplanetary Dust Grains
}

\author{
Hajime Okamoto \\ The Graduate School of Science and Technology, Kobe University, \\ Nada, Kobe 657, Japan.
}

\begin{abstract}
The first scattering coefficient (a1-term) in Mie theory is introduced to determine the dipole polarizability used in the discrete dipole approximation (DDA) to calculate the scattering properties of the cluster of spheres in order to improve our understanding of interplanetary dust particles. In this method, each sphere in a cluster is replaced by a single dipole. The accuracy of this method is tested for a few spheres in contact. It is confirmed that the al-term method is superior to other types of DDA and is particularly suitable for the case when the particles are placed randomly. By using this method, it becomes possible to treat large cluster of spheres, i.e., a size parameter of the target $X\left(\equiv 2 \pi r_{e q} / \lambda\right) \sim$ 50 , where $r_{e q}$ is a volume equivalent radius of the target, and $\lambda$ is the wavelength of incident wave.
\end{abstract}

\section{Introduction}

The discrete dipole approximation (DDA), originally developed by Purcell \& Pennypacker(1973), has been applied to variety of problems. This is because, in this method, the continuum target is replaced by an array of point dipoles, therefore it may be easily adapted to any target geometry. However due to a large memory requirement and long computing time, the applicability of the DDA is restricted to relatively small particles. The DDA works well with volume size parameter $X \leq 10$ (Draine\&Goodman1993). That is, the applicability of the DDA is restricted to $r_{e q} \leq 1 \mu \mathrm{m}$ at the visible wavelength. In this paper, we have tried to overcome this practical problem in the DDA calculations by changing the description of the dipole polarizability.

\section{Several choices of dipole polarizabilty in the DDA}

In the DDA calculations, it is necessary to determine the polarizabilities of the point dipoles. Draine(1988) improved the Clausius-Mossotti relation by introducing radiative reaction corrections (hereafter CMRR). Goedecke \& O'Brien (1988) and Hage \& Greenberg (1990) proposed Digitized Green's Function or Volume Integral Equation Formulation (hereafter DGF/VIEF) to define the polarizabilities. On the other hand, Draine\&Goodman(1993) derived the Lattice Dispersion Relation (LDR) in order to obtain dipole polarizabilities for a cubic array. They have shown that LDR representation is superior to the others when 
a sphere was chosen as a target. According to the Mie theory, we may find another relation to set the dipole polarizability. That is, by using a scattering coefficient $a_{1}$ term, the dipole polarizability $\alpha$ is written as (Doyle 1989)

$$
\begin{gathered}
\alpha=i \frac{3 a_{1}}{2 k^{3}} \\
a_{1}=\frac{m \psi_{1}\left(m X_{m}\right) \psi_{1}^{\prime}\left(X_{m}\right)-\psi_{1}\left(X_{m}\right) \psi_{1}^{\prime}\left(m X_{m}\right)}{m \psi_{1}\left(m X_{m}\right) \xi_{1}^{\prime}\left(X_{m}\right)-\xi_{1}\left(X_{m}\right) \psi_{1}^{\prime}\left(m X_{m}\right)},
\end{gathered}
$$

where $\psi_{1}$ and $\xi_{1}$ are the first order Riccati-Bessel functions and $X_{m}$ denotes the size parameter of the spherical monomer, i.e., $X_{m}$ is defined by $k r_{m}$ and $r_{m}$ is the radius of the sphere. When we use the al-term method described above, a spherical monomer in the cluster is replaced by a single dipole. And the dipole polarizability of the monomer is determined by the a1-term. The accuracies of this method were reported in Okamoto (1995) for some particular orientations. Here the estimate of the error for random orientations will be provided.

\section{Results and Discussions: The accuracies of the a1-term method}

We have compared the accuracies of the results obtained using the a1-term method with those by several previous methods, i.e., CMRR, DGF/VIEF and LDR for a few spheres in contact. In each DDA model, the sphere is replaced by a single dipole. For a homogeneous sphere, Mie theory can be used to derive the relative errors in the DDA results. It is found that the a1-term method gives the most accurate results as long as the size parameter of this sphere is smaller than 2. The errors for the LDR, where $\mathrm{N}=1$ dipole is used to represent the target, become unacceptably large $(>100 \%)$ while those for the a1-term are $5 \%$, when the refractive index $m=1.33+0.01 i$ is considered. This comes from the fact that the boundary condition of the sphere is taken into account in the a1-term method while the LDR is optimized for the cubic unit cell.

A non-spherical particle has also been considered as a scattering target to test the accuracies of the a1-term method. For the results by the a1-term nnethod. two dipoles have been used. Besides the a1-term method we used the LDR. For two-touching spheres the size parameter of this sphere is ranging from 0.2 to 2 . For the results derived by the LDR, several different sets of number of dipoles $N$ have been used to represent the target, i.e., $N$ is ranging from 2 to 34512 . In the $N=34512$ model, each sphere in the cluster is replaced by 17256 dipoles. We found that the solutions for $N=8448$ dipoles with the LDR in the cluster are nearly the same as those for $N=34512$ for three independent orientations of the target. Therefore it appears that the solutions for $N>8000$ with the LDR are the same. On the basis of the above results, the relative errors of each model were derived by a comparison to the DDA results for $N=8448$ for the two-touching spheres when the target is oriented randomly. Fig. 1 describes the relative errors in the extinction efficiencies $Q_{\text {ext }}$ for the a1term method' and Fig. 2 described those in the scattering efficiencies $Q_{\text {sca }}$. It is confirmed that for $N=2$ the LDR model significantly overestimates the extinction efficiencies when the size of the monomer in the cluster is comparable to the wavelength but the a1-term method does not. Within the accuracy of $5 \%$ 


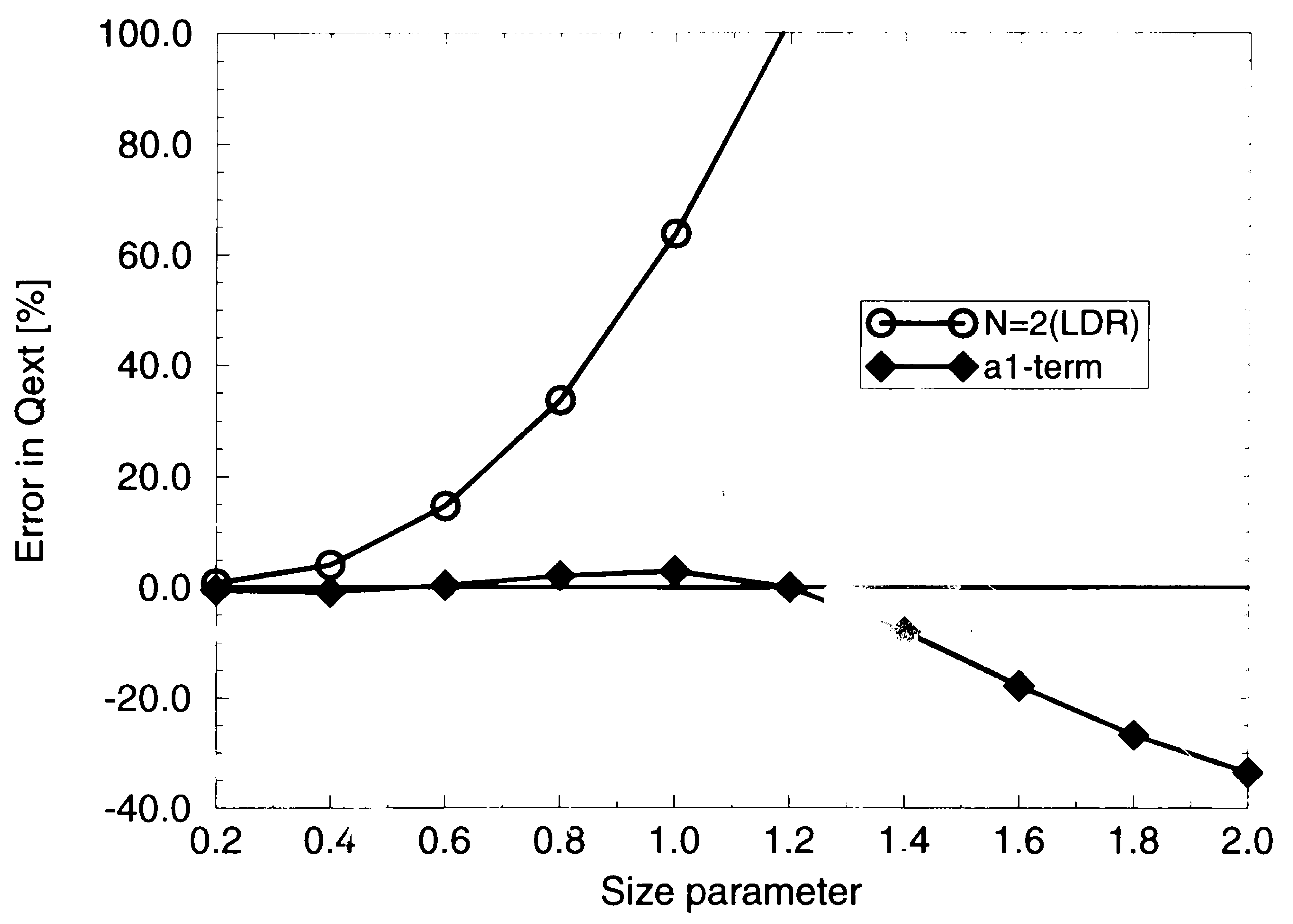

Figure 1. Relative errors in extinction efficiencies for the a1-term method and the LDR, where two dipoles are used in the DDA. Twotouching spheres with refractive index $m=1.33+0.01 i$ is chosen.

in $Q_{\text {ext }}$ and that of $10 \%$ in $Q_{s c a}$, it is possible to treat relatively large monomers with $X_{m} \sim 1$ (corresponding to $X=1.3$ ) in the a1-term method. Therefore, we can expect that the a1-term method is especially suitable for the scattering and extinction calculations when the particles are assumed to be oriented randomly which might be of particular interest in interplanetary dust studies. It should be noted that recently it becomes possible to derive the general solution for cluster of several spheres (see Fuller 1991, Mackowski 1991 and Xu 1995). Flatau et al.(1993) actually compared the DDA results with this multipole solutions for two-touching spheres. They showed that the results for $N=34512$ dipoles are quite accurate, i.e. the relative errors in $Q_{s c a}$ and $Q_{a b s}$ are around $1 \%$ as long as $X \leq 10$ and $m=1.33+0.01 i$. Therefore their conclusion supports our error analysis.

In conclusions, the a1-term method used here allows to treat relatively large sub-volume element which is replaced by a dipole, i.e. the size parameter of the monomer $X_{m} \sim 1$ which corresponds to $r_{m}=0.1 \mu \mathrm{m}$ at visible wavelength. Consequently, when we use $N \sim 10^{5}$ dipoles, the DDA calculations based on the a1-term method is now feasible for the cluster with the size parameter $X \sim 50$ which corresponds to $r_{e q} \sim 5 \mu \mathrm{m}$, i.e. five times larger than the maximum size parameter when the LDR is used. It should be noted the applicability of the a1-term is restricted to the clusters. We can apply this method for the cluster of spheroids by introducing the first scattering term from the spheroid theory 


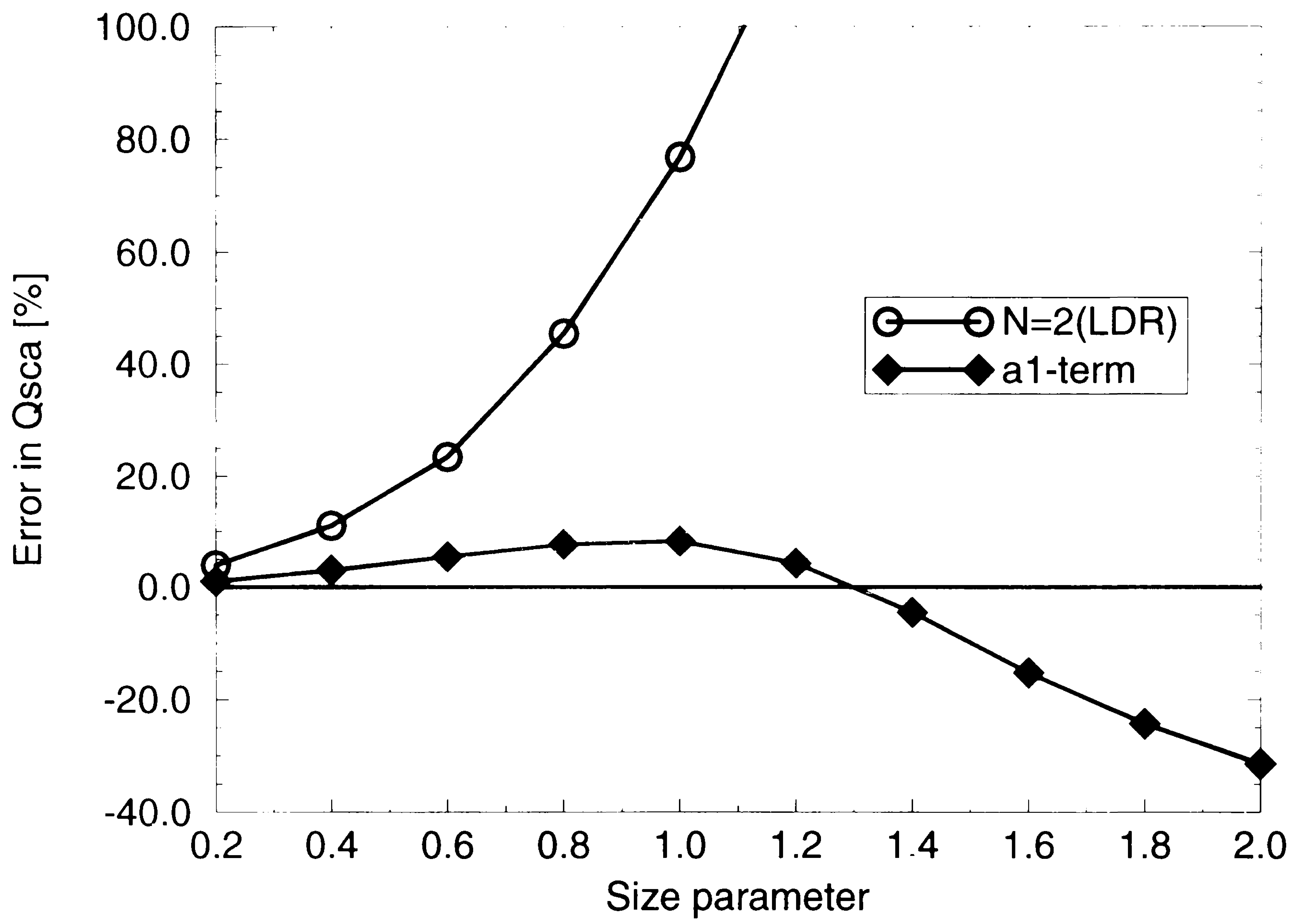

Figure 2. The same as Fig.1 but for scattering efficiencies.

proposed by Asano \& Yamamoto (1975) and also for clusters of monomers which have core-mantle structure.

Acknowledgments. We thank B. Draine \& P Flatau for providing the original DDA codes, and T. Mukai \& B. Draine for discussions.

\section{References}

Asano. S. \& Yamamoto,.G. 1975, Appl. Optics, 14, 29

Doyle. W. T 1989, Phys. Rev. B, 39, 9852

Draine, B. T 1988, ApJ. 333. 848

Draine, B. T \& Goodman, J 1993, ApJ, 405, 685

Flatau. P J Fuller, K.A. \& Mackowski, D. W 1993, Appl. Optics, 32, 3302

Fuller, K. A. 1991, Appl. Optics, 30,4716

Goedecke. G. H. \& O’Brien, S. G. 1988, Appl. Optics, 27,2431

Hage. J. I. \& Greenberg, J M.1990, Appl. Optics, 14,29

Mackowski. D. W. 1991, Proc. R. Soc. Lond. A., 433, 599

Okamoto. H. 1995. Opt. Rev., 6, 407

Purcell. E. M. \& Pennypacker, C. R. 1973, ApJ, 186, 705

Xu. Y 1995. Appl. Optics, 34,4573 